\title{
Localized and Programmable Chemical Vapor Deposition Using an Electrically Charged and Guided Molecular Flux
}

\author{
Johannes Reiprich ${ }^{1}$, Nishchay A. Isaac ${ }^{1}$, Leslie Schlag1, Thomas Kups², \\ Marcus Hopfeld², Gernot Ecke ${ }^{1}$, Thomas Stauden ${ }^{1}$, Jörg Pezoldt ${ }^{1}$ and Heiko \\ O. Jacobs ${ }^{1 *}$
}

\footnotetext{
${ }^{1}$ Fachgebiet Nanotechnologie, , Institut für Werkstofftechnik, Institut für Mikro- und Nanoelektronik und Institut für Mikro- und Nanotechnologien MacroNano®, Technische Universität IImenau, Postfach 100565, 98684 IImenau, Deutschland

${ }^{2}$ Fachgebiet Werkstoffe der Elektrotechnik, Institut für Werkstofftechnik, Institut für Mikro- und Nanoelektronik und Institut für Mikro- und Nanotechnologien MacroNano®, Technische Universität IImenau, Postfach 100565, 98684 IImenau, Deutschland

*E-mail: heiko.jacobs@tu-ilmenau.de
}

\section{Supporting Information}

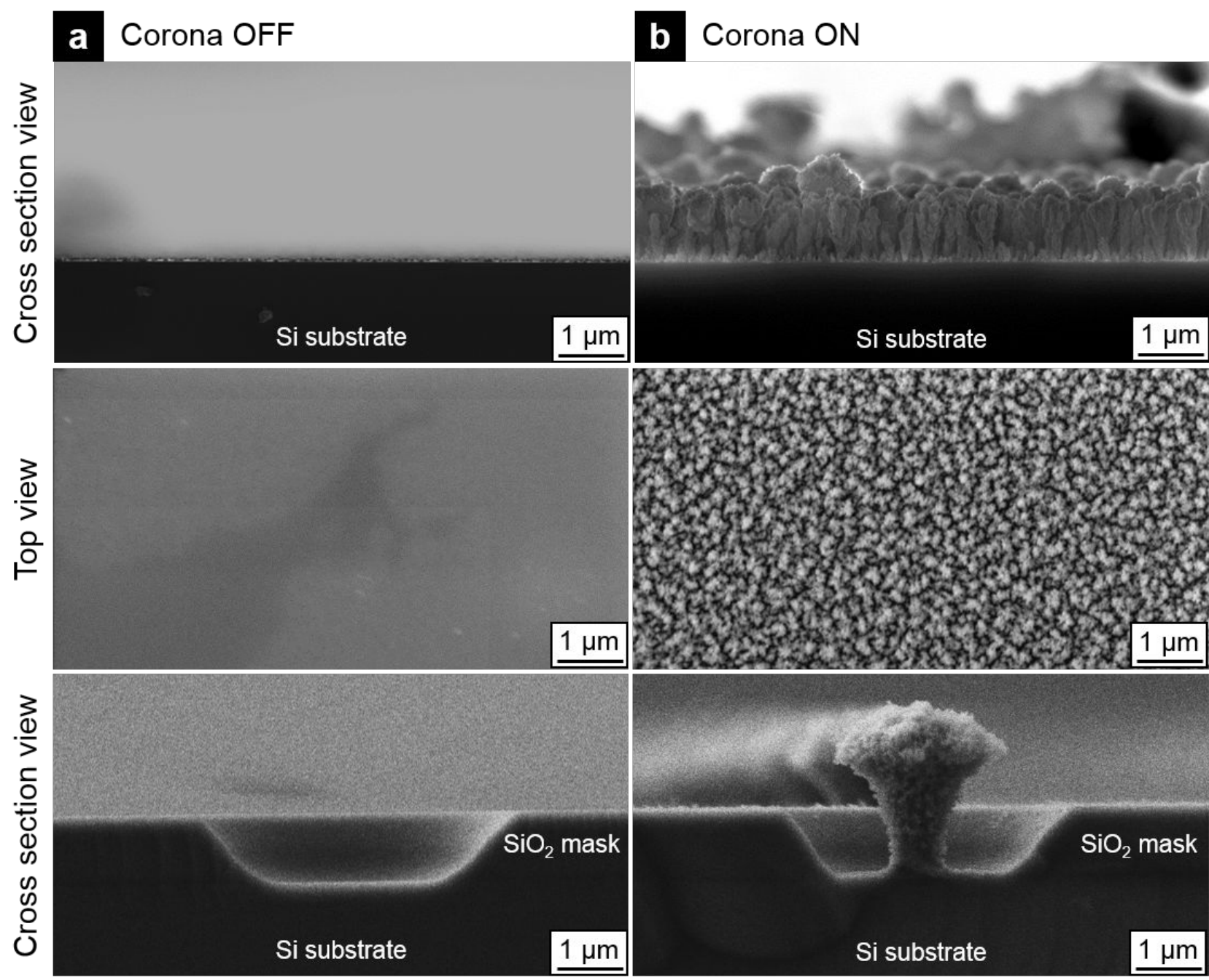

Figure S1 Comparison of the deposition rate without (a) and with (b) charged molecular flux. Copper oxide deposition was performed on unmasked (first two rows) and masked (last row) silicon substrates. The magnification is the same in all SEM images. When comparing the left and the right images, it becomes clear, that the deposition rate increases when the corona discharge is switched on. The corona discharge charges 
the precursor molecules and they drift towards the grounded substrate. This leads to a higher deposition rate. For unmasked substrates, the average layer height is $0.1 \mathrm{~nm}$ without corona discharge and $3.1 \mu \mathrm{m}$ with corona discharge (first row). The average surface coverage is $3.6 \%$ without corona discharge and $96.4 \%$ with corona discharge (second row). When the substrate is structured and the corona is switched on, local deposition occurs (last row). The deposition rate without corona discharge varies between 0 (not measureable) and 0.5 $\mathrm{nm} / \mathrm{min}$ and the deposition rate with corona discharge varies between 80 and $125 \mathrm{~nm} / \mathrm{min}$. By combining the layer height and surface coverage, the material transport can be calculated. With corona discharge, the material transport is increased by a factor of 12700. The values were acquired by 8 individual measurements. 


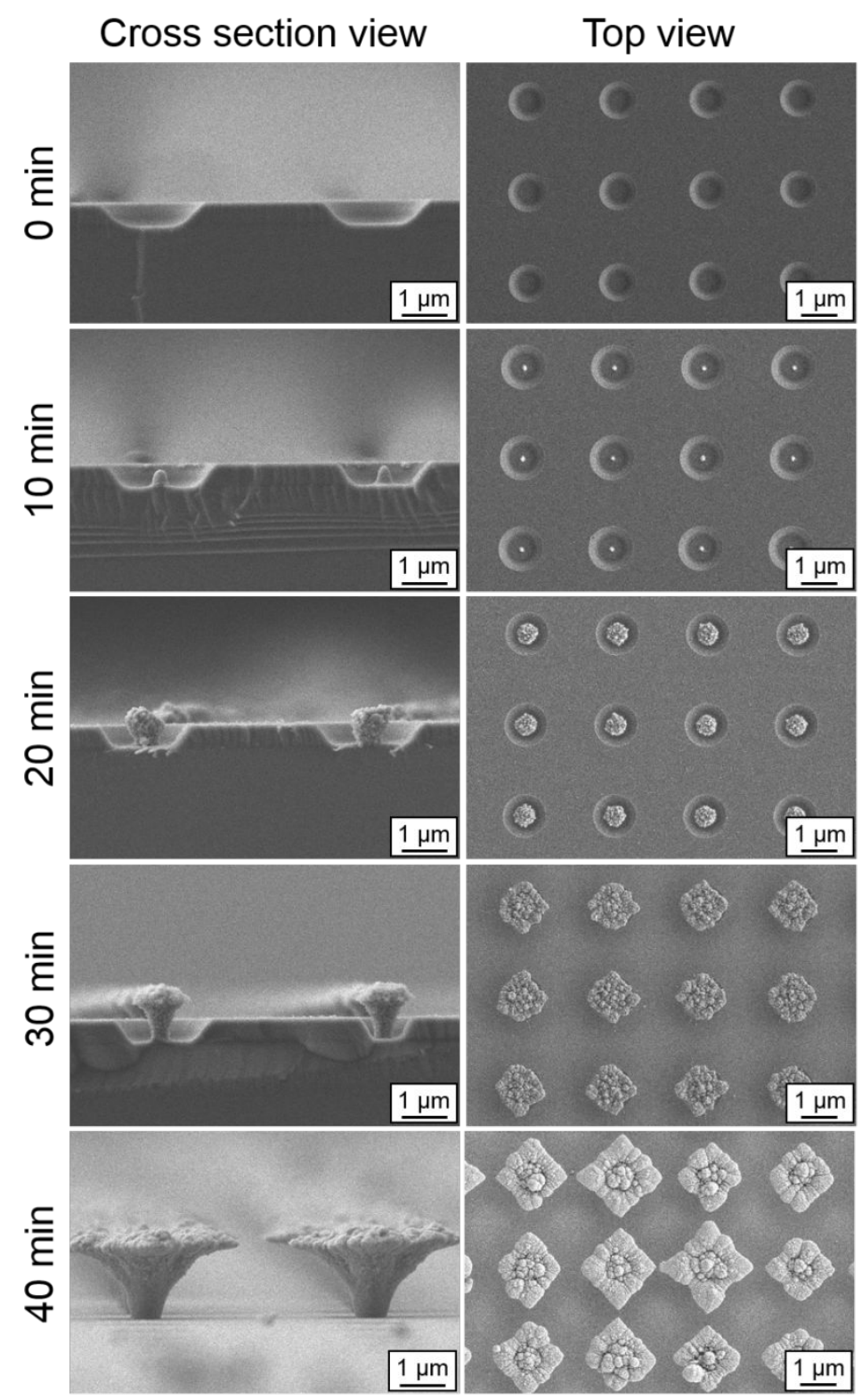

Figure S2 SEM images of the localized deposition of copper oxide in cross section (left column) and top view (right column). The deposition was conducted for different times to observe the evolution of the structures. No deposition was conducted on the reference substrate $(0 \mathrm{~min})$. The molecular flux focuses the precursor molecules in the center of the openings. This is why the deposition starts with a circular structure in the center (10 min). The structure is getting taller with increased process time $(20 \mathrm{~min})$. After $30 \mathrm{~min}$, the copper oxide structures start to spread out of the openings and they start to align to their next neighbors. After 40 min process time, the structures nearly touch their neighboring structures. The shape of the deposited structures is given by the mask design and the present electric fields. 


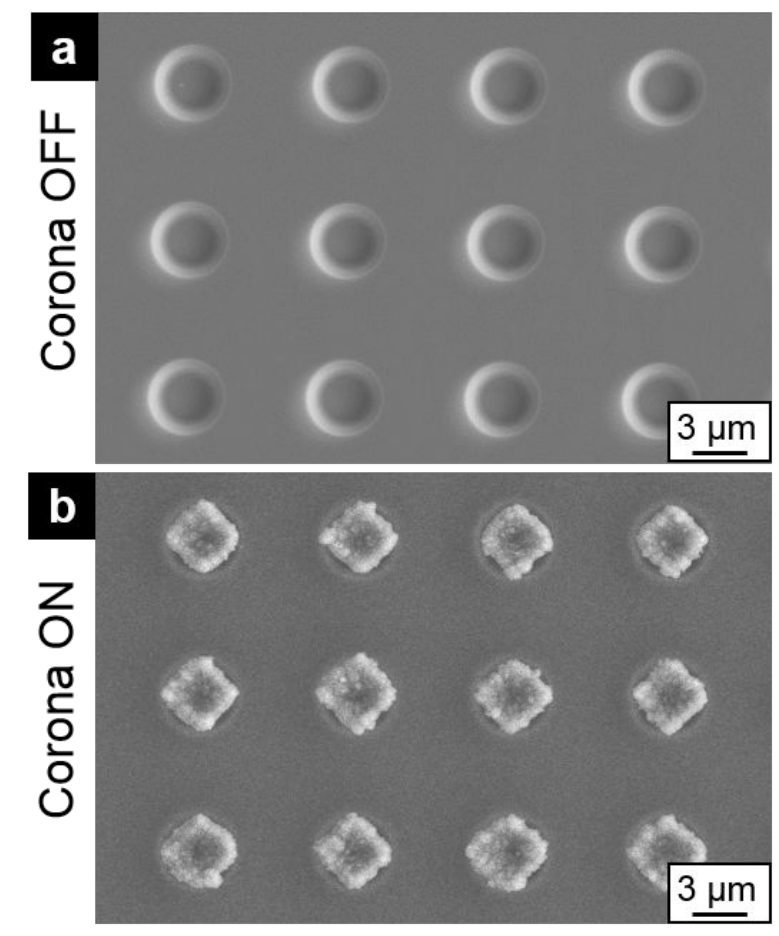

Figure S3 Comparison of the deposition without and with corona charger. SEM images of 12 deposition locations. Deposition was conducted (a) without igniting the corona discharge and (b) with corona discharge ignited. Without corona charger, no deposition can be observed. When the corona discharge is ignited instead, localized deposition occurs. The redirection of the material flux towards the deposition locations leads to a higher deposition rate in these areas compared to the surrounding areas. 

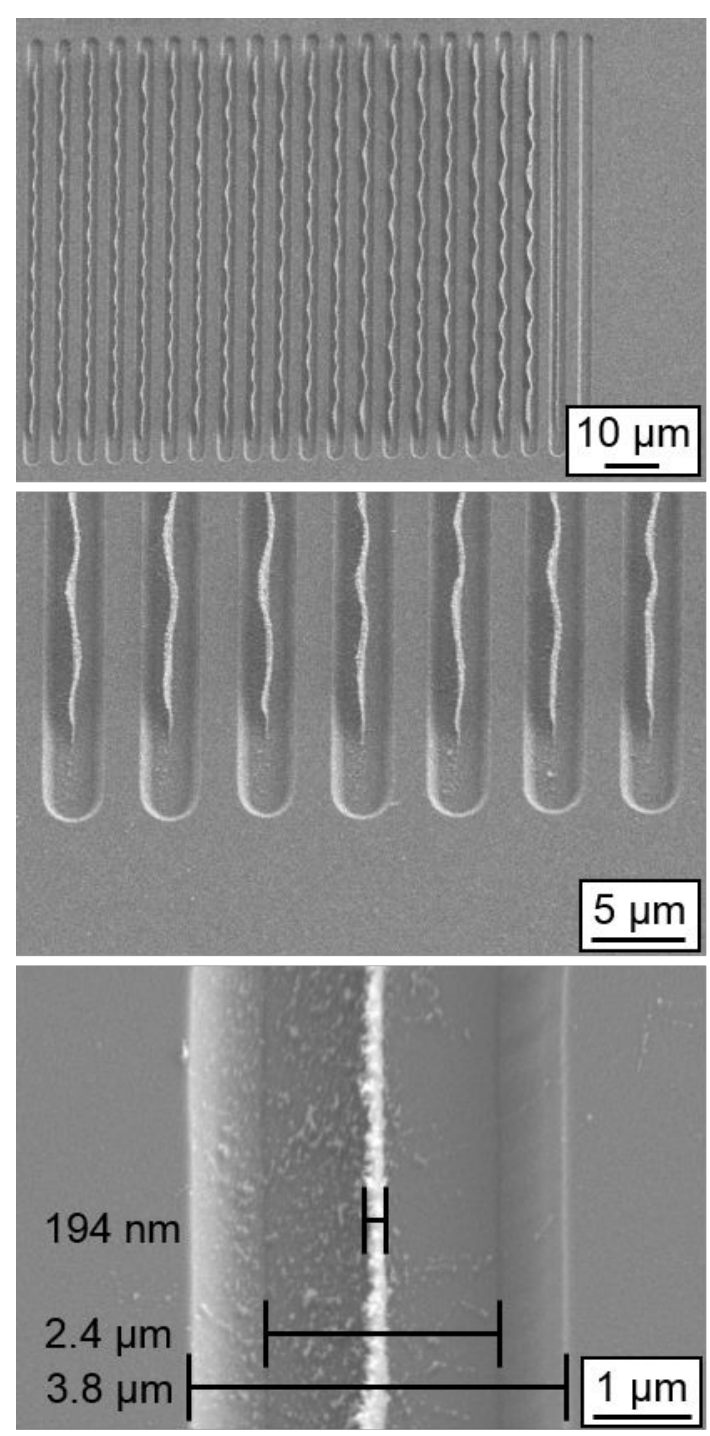

Figure S4 SEM images of localized deposition of copper oxide in parallel lines. The three SEM images are acquired with three different magnifications. The charged molecular flux focusses the precursor molecules inside the openings, leading to smaller copper oxide structures than the mask. The mask openings are $2.4 \mu \mathrm{m}$ and the minimum feature size is $194 \mathrm{~nm}$. 
a
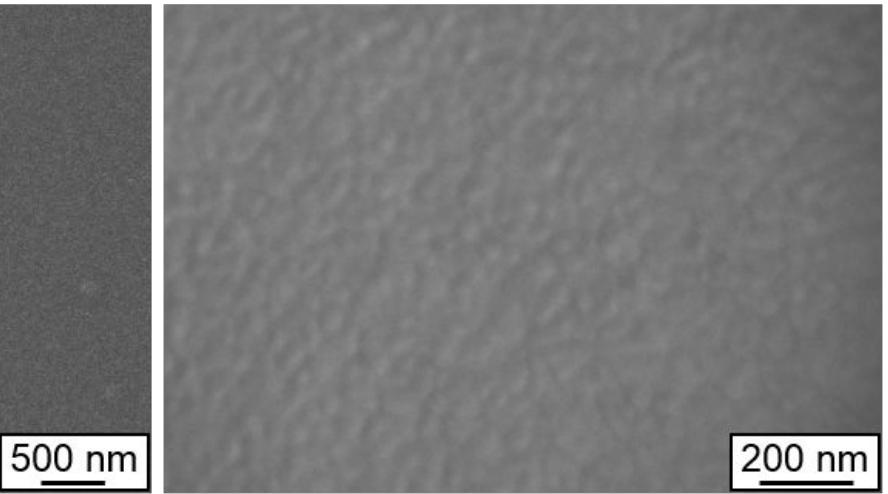

b

$500 \mathrm{~nm}$

$200 \mathrm{~nm}$

Figure S5 SEM images of an opening in the $\mathrm{SiO}_{2}$ towards the underlying gold layer (a) before and (b) after conducting a typical deposition process for copper oxide. To investigate the influence of the gold, the charged molecular flux was not used (corona discharge turned off). By comparing the openings before and after the growth, no catalyzing effect of the gold could be observed. The gold remains unchanged during the deposition process $\left(30 \mathrm{~min}\right.$ and $\left.260^{\circ} \mathrm{C}\right)$. 


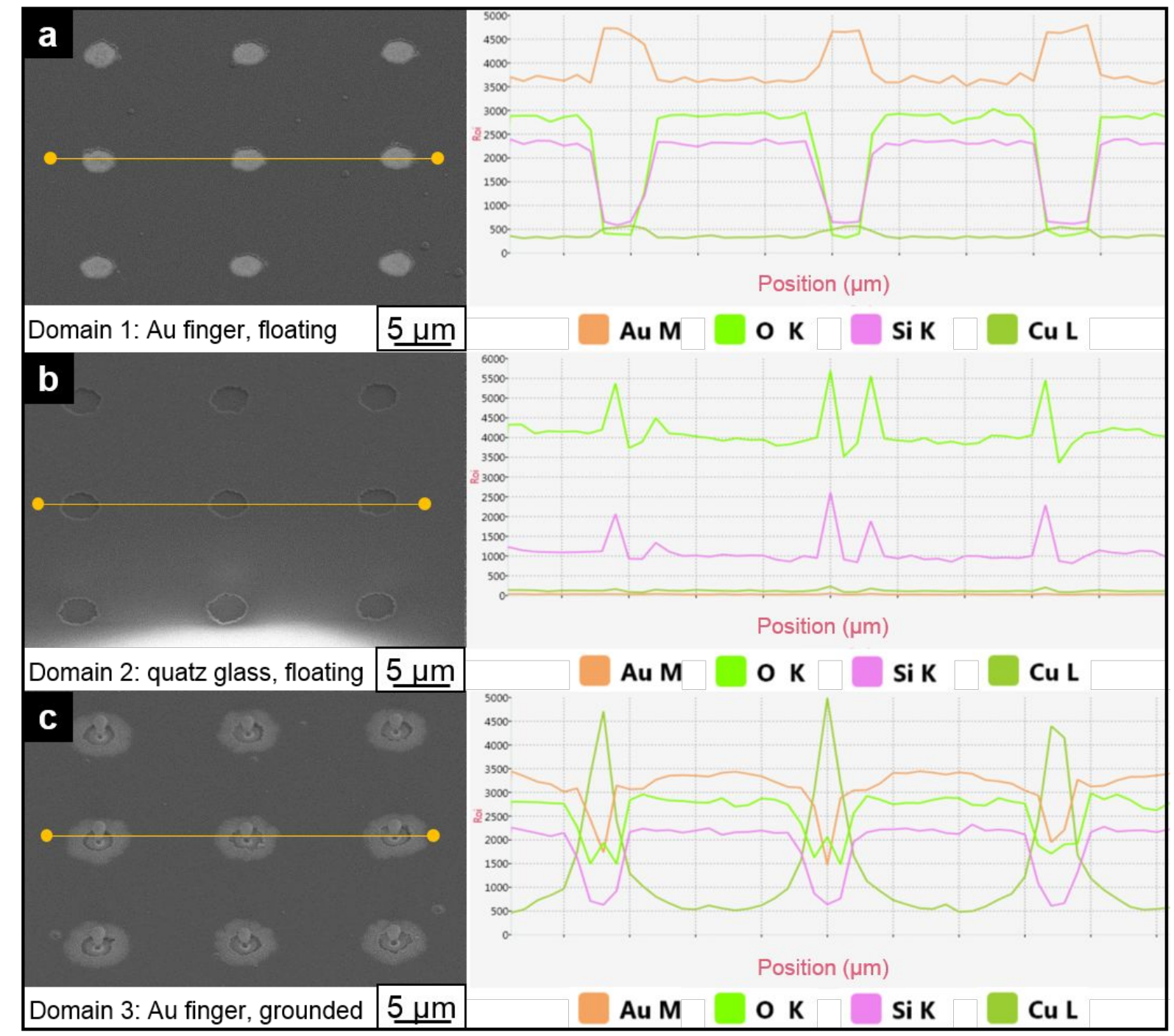

Figure S6 EDX line scan across different deposition domains. SEM images of three different deposition domains on a substrate are presented: (a) a gold domain which is not connected electrically floating potential), (b) a quartz glass domain (floating potential), and (c) a gold domain which is connected to ground potential. Next to the SEM images, corresponding EDX line scans are presented. The yellow line in the SEM images presents the path the EDX line scan follows. Along this line, the intensity of the Au M, the OK, the Si $K$ and the $C u L$ line is measured and plotted against the position on the substrate. The Au M line stems from the gold of the electrode, the $\mathrm{Si} \mathrm{K}$ line from the $\mathrm{SiO}_{2}$ mask, and the $\mathrm{Cu} L$ line from the deposited copper oxide. On the one hand, the $\mathrm{O} K$ line belongs to the $\mathrm{SiO}_{2}$ and on the other hand to the copper oxide. In (a), the Au intensity is increased when scanning over a deposition location, whereas the Si and O intensity is decreased. The deposition locations are designed as holes in a $\mathrm{SiO}_{2}$ mask towards a gold electrode. The EDX line scan reproduces this design. In (b), only Si and $\mathrm{O}$ can be found as this domain only consist of glass. In (c), the $\mathrm{Cu}$ intensity is increased at the deposition locations. This proves the localized deposition of copper oxide. 

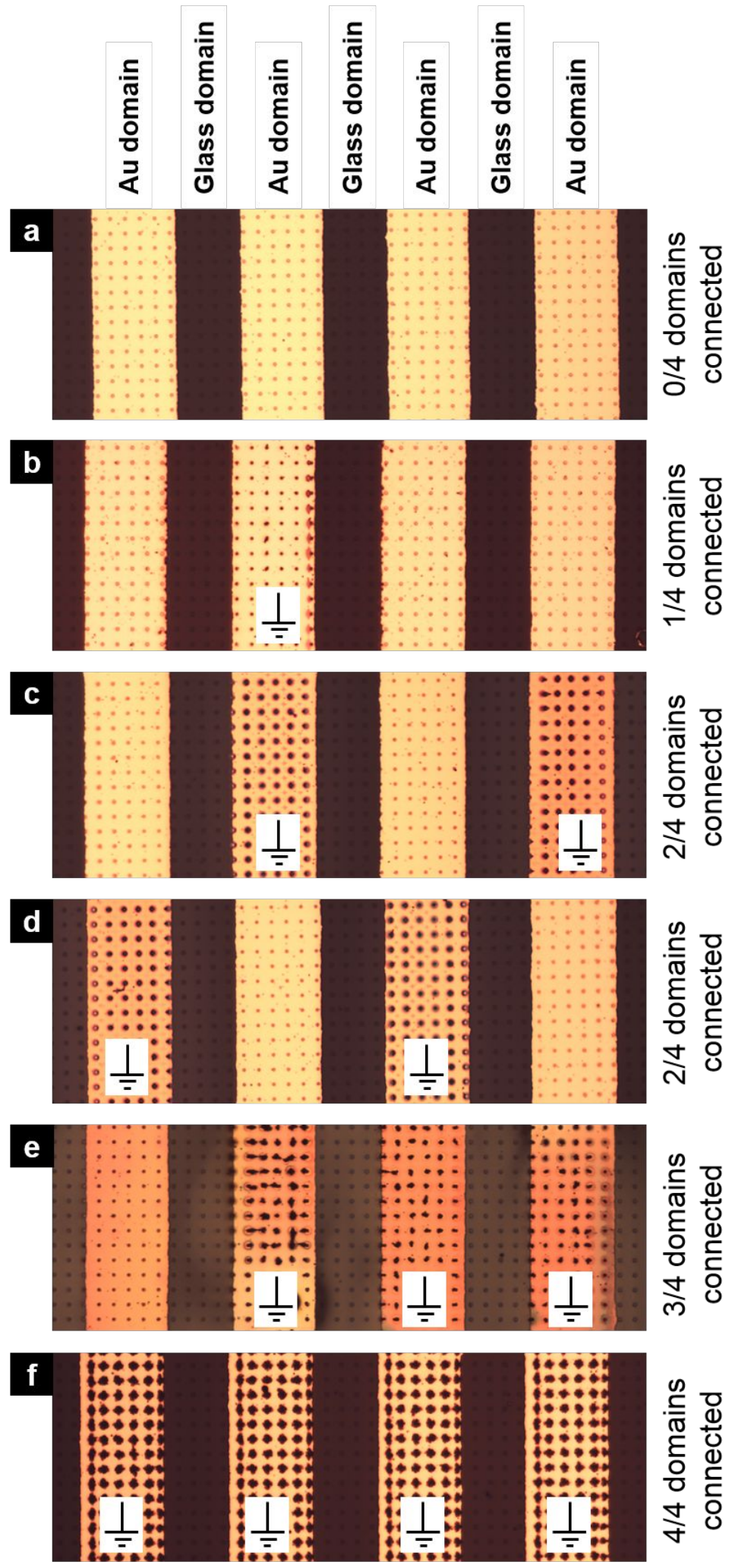

Figure S7 Four individual domain electrodes. Microscopy images of localized and programmable CVD of copper oxide on four individual domain electrodes. Deposition was conducted with different biasing schemes: (a) no electrode was connected during deposition, (b) one out of four electrodes was connected, (c and d) two out of four electrodes were connected, (e) three out of four electrodes were connected, (f) all electrodes were connected. It can be proven, that deposition only occurs on electrodes which are electrically connected. On the other electrodes, no deposition occurs. In all cases, no deposition occurs in the quartz glass domains which are insulating. 